attempt to distinguish between the two and repeatedly uses complex as the opposite of real. In a book on complex geometry this is especially to be regretted.

The book is marred by many, very many, typographical errors. Those in the text itself are not seriously disturbing; in fact, some of them, as, for example, "any value linearly descendent on two given values" on p. 108, and "two intersectional minimal lines" on p. 190, are decidedly the opposite. But those in the formulas, though they may be only irritating to the mature reader, are likely to prove a stumbling block for the budding mathematician.

Aside from these matters, the book is full of solid, stimulating mathematics. Moreover, it is particularly welcome in that it brings up to date a field of geometry which is comparatively new and by no means exhausted. May it prove the inspiration and basis of departure for fresh endeavors.

W. C. Graustein

\title{
GAUSS AND NON-EUCLIDEAN GEOMETRY
}

A question of historical accuracy is raised by Professor Emch in his review of my Projective Geometry* where he says:

"It is proper to point out an error which is common in histories of mathematics and which is contained in the following statement on page 420: 'Little progress was made until about a hundred years-later when Gauss (1777-1855), his friends and pupils became deeply interested in the subject.' (1) Now the fact is that Gauss's deeper interest in the subject was subsequently aroused by the brilliant discoveries of Lobatchevsky and Bolyai. (2) As a matter of fact, Gauss, in the beginning, hoped to be able to prove what is known as the euclidean parallel axiom and (3) assumed a rather skeptical attitude towards the new discoveries. (4) Subsequent deeper meditations, however, led Gauss to his own establishment or verification and acceptance of the new theory." +

An examination of the sources on which my statement is based will, I believe, substantiate the sentence quoted as well as the context from which it is taken. Among these sources are the letters of the

* This Bulletin, vol. 30 (1924), p. 81.

$\dagger$ The numbering is mine. 
two Bolyais and Gauss himself.* of the four points in Professor Emch's own statement, (2) is correct but irrelevant. For it applies equally to all the pioneers in the subject, including Lobachersky and young Bolyai too t who may be said to have inherited the problem of parallels from his father, Wolfgang Bolyai.

In Gauss's works (loc. cit.) are found some twenty letters dealing. with the foundations of geometry and the theory of parallels, the first written in 1799. From these we learn that he had attacked the problem as early as 1792 (letter to Schumacher, 1846) while he first learned of the work of John Bolyai when he received from the father a copy of the famous Appendix in 1832 (letter to Gerling). $\$$ Prior to this time he expresses his doubts concerning the validity of Euclid (letter to W. Bolyai, 1799) and states his conviction that "the necessity of our geometry cannot be proved" and hence "we must place geometry somewhat in the category with mechanics" (letter to Olbers, 1817). $\mathrm{He}$ obtained many of the fundamental properties of the hyperbolic geometry, e. g. the impossibility of similar figures, the existence of an absolute a priori measure of length (letter to Gerling, 1816), the connection between the defect of the angle sum and the area of a triangle, the formula for the area of the maximum triangle (letter to Gerling, 1819) and the formula for the length of a circle (letter to Schumacher, 1831). He even originated the name non-euclidean geometry, declared it to be self-consistent throughout and that it reduced to the euclidean when the space constant is infinite (letter to Taurinus, 1824). Various reasons are assigned for not publishing his results, such as the lack of time for refining them, the indifference and incompetence of the mathematical public and the anticipated "clamor of the Boeotians".

This seems to me ample justification of my statement that Gauss was deeply interested in the problem of parallels and had made sub-

* Stäckel and Engel, Gauss, die beiden Bolyai und die nichteuklidische Geometrie, Mathematische Annalen, vol 49 (1897), p. 149; Gauss, Werke, vol. 8, pp. 159-239. Many of these are available in English, e. g. in Carslaw, Non-Euclidean Geometry, pp. 19-37.

+ Three proofs of the parallel postulate by Lobachersky are contained in notes of his lectures (1815-1817) and another apparently in a paper, now lost, but read at Kasan in 1826, entitled Exposition succincte des principes de la géometrie, avec une démonstration rigoreuse du théorème des parallèles.-Carslaw, loc. cit. John Bolyai was seeking a proof of the postulate at about 1820 according to a statement in his autobiography, quoted by Stäckel and Engel, loc. cit., p. 155.

$\mp$ He appears not to have heard of the work of Lobachevsky until some years later (letter to Encke, 1841) and notes by Stäckel. 
stantial progress toward its solution prior to his knowledge of the work of J. Bolyai and Lobachevsky. It would be interesting, if space permitted, to listen at length to Gauss's own testimony but a few extracts from his letters will have to suffice:

1. To Schumacher, May 17, 1831. "Some of my own meditations which in part are now about 40 years old-whereof however I have never recorded anything and therefore have been obliged to think out many of them three or four times anew-I have begun in the past few weeks to write down. For I did not wish it to perish with me."

2. To Gerling, February 14, 1832 . ... "I have this. day received from Hungary a little treatise on the Non-Euclidean geometry wherein I find again all my own ideas and results, developed with great elegance, howbeit for one to whom the subject is strange, in a form somewhat difficult to follow because of the concentration. ... I regard this young geometer von Bolyai as a genius of the first order."

3. To Wolfgang Bolyai, March 6, 1832 (in response to a letter accompanying the Appendix by John Bolyai). ... "If I should begin by saying that I am unable to praise it, you would doubtless be astonished for a moment. But I cannot do otherwise-to praise it would be to praise myself. For the whole content of the work, the method which your son has employed and the results to which he is led, agree almost completely with my own meditations pursued in part now for 30-35 years." ...*

4. To Schumacher, November $28,1846 . \quad \ldots$ "I have recently had occasion to examine again the little work by Lobatschewsky (Geometric Researches on the Theory of Parallels, Berlin, 1840). It contains the elements of that geometry which must exist and with strict consistency may exist if the euclidean geometry is not the true one. A certain Schweikart called such a geometry astral geometry, Lobatschewsky imaginary geometry. You know that for 54 years (since 1792) I have held the same conviction (with a certain later extension which I shall not mention here). I have not found therefore in Lobatchevsky's work material new to me but the development is made on other lines than I have followed and indeed by Lobatchevsky in amasterly manner with true geometric genius." ...

R. M. WINGER

* John Bolyai was only 29 years old when this was written. 